\title{
HOLOMORPHIC MOTIONS AND POLYNOMIAL HULLS
}

\author{
ZBIGNIEW SLODKOWSKI
}

(Communicated by Paul S. Muhly)

\begin{abstract}
A holomorphic motion of $E \subset \mathbb{C}$ over the unit disc $D$ is a map $f: D \times \mathbb{C} \rightarrow \mathbb{C}$ such that $f(0, w)=w, w \in E$, the function $f(z, w)=f_{z}(w)$ is holomorphic in $z$, and $f_{z}: E \rightarrow \mathbb{C}$ is an injection for all $z \in D$. Answering a question posed by Sullivan and Thurston [13], we show that every such $f$ can be extended to a holomorphic motion $F: D \times \mathbb{C} \rightarrow \mathbb{C}$. As a main step a "holomorphic axiom of choice" is obtained (concerning selections from the sets $\left.\mathbb{C} \backslash f_{z}(E), \quad z \in D\right)$. The proof uses earlier results on the existence of analytic discs in the polynomial hulls of some subsets of $\mathbb{C}^{2}$.
\end{abstract}

\section{INTRODUCTION AND STATEMENT OF RESULTS}

Holomorphic motions are isotopies depending holomorphically on a complex parameter. Their study was originated by Mañé et al. [8], in the context of the dynamics of rational maps, and was continued by Sullivan and Thurston [13] and Bers and Royden [2].

Definition [13]. Let $E$ be a subset of $\mathbb{C}$. A holomorphic motion of $E$ in $\mathbb{C}$, parametrized by the unit disc $D$, is a map $f: D \times E \rightarrow \mathbb{C}$ such that (a) for any fixed $w \in E$, the map $z \rightarrow f(z, w): D \rightarrow \mathbb{C}$ is holomorphic; (b) for any fixed $z \in D$, the map $w \rightarrow f(z, w)=f_{z}(w)$ is one-to-one; and (c) $f_{0}$ is the identity map on $X$.

Note that no continuity in $w$ or $(z, w)$ is assumed here. However, it holds due to the following remarkable "lambda lemma" of Mañé et al.

Lemma 1.1 [8]. If $f: D \times E \rightarrow \mathbb{C}$ is a holomorphic motion, then $f(z, w)$ is jointly continuous and has a continuous extension to $F: D \times \bar{E} \rightarrow \mathbb{C}$. Furthermore, $F$ is a holomorphic motion of $\bar{E}$ over $D$, and the injections $F_{z}(\cdot)=$ $F(z, \cdot)$ are quasiconformal.

Received by the editors January 5, 1989; presented at the AMS Special Session in Complex Analysis, Fayetteville, Arkansas, March 24, 1990.

1980 Mathematics Subject Classification (1985 Revision). Primary 30E25, 32E20; Secondary 30D45, 30C60.

Key words and phrases. Holomorphic motion, isotopy, polynomially convex hull, analytic disc, hyperbolic domain.

This research was supported in part by National Science Foundation grant DMS-8901861. 
If we consider the "trace" of holomorphic motion, i.e., the set

$$
\left\{\left(z, f_{z}(w)\right): z \in D, w \in E\right\} \subset D \times \mathbb{C},
$$

we observe that it is foliated by a family of analytic discs. (An analytic disc $=$ the graph of an analytic map $D \rightarrow \mathbb{C}$.) A similar property was obtained for polynomially convex hulls of some classes of subsets of $\mathbb{C}^{2}$ by Alexander and Wermer [1], by Forstneric [3], and by the author [11, 12]. We quote next the most general of those results. (Below, $h(X)$ denotes the polynomial hull of the set $X$.)

Theorem 1.2 [12]. Let $X \subset \partial D \times \mathbb{C}$ be a compact set such that the fibers $X(\zeta):=$ $\{w \in \mathbb{C}:(\zeta, w) \in X\}$ are connected for all $\zeta \in \partial D$. Assume $h(X) \cap D \times \mathbb{C} \neq \varnothing$. Then, $h(X) \backslash X$ is the union of a family of analytic discs. Furthermore, the topological boundary $S$ of $h(X) \backslash X$ in $D \times \mathbb{C}$ is the union of a family of mutually disjoint analytic discs.

An obvious reinterpretation of this result in terms of holomorphic motion is that the part $S$ of the boundary of the hull $h(X)$ is the trace, in the sense of (1.1) of some holomorphic motion (unique, in fact) of the fiber $S(0)$. (We will also see in $\S 3$ that the whole set $h(X) \backslash X$ is the trace of some holomorphic motion.) The purpose of this paper is to use the relation between polynomial hulls and holomorphic motions to prove Theorems 1.3 and 1.4 below, answering thereby two questions posed by Sullivan and Thurston [13].

The first concerns the extendability of holomorphic motions.

Theorem 1.3. Every holomorphic motion $f: D \times E \rightarrow \mathbb{C}$ of an arbitrary subset $E$ of $\mathbb{C}$ can be extended to a holomorphic motion $F: D \times \mathbb{C} \rightarrow \mathbb{C}$ (that is $F \mid D \times E=f)$ of $\mathbb{C}$, parametrized by the same unit disc $D$.

Partial extension results already have been reached. Sullivan and Thurston [13] proved the existence of $F: D(0, r) \times \mathbb{C} \rightarrow \mathbb{C}$, where $D(0, r)=\{z:|z|<r\}$ and $r$ is a positive and uniform, but otherwise unspecified, constant. Bers and Royden [2] gave another construction in which $r=\frac{1}{3}$. Our result amounts to showing that $r=1$; in addition, the methods are different from those of [2] and [13], and are, arguably, simpler.

The next theorem asserts another conjecture of Sullivan and Thurston [13], called by them the "holomorphic axiom of choice."

Theorem 1.4. Let $f(z, w)=f_{z}(w)$ be a holomorphic motion of a subset $E \subset$ $\mathbb{C}$, parametrized by $z \in D$. Then, for every point a outside $E$, there is a holomorphic map $g: D \rightarrow \mathbb{C}$ such that

(i) $g(0)=a$ and

(ii) $g(z) \notin f_{z}(E)$ for every $z \in D$.

Since it has already been indicated by Sullivan and Thurston [13] that (and how) the holomorphic axiom of choice would imply the extension result, our main effort is directed toward obtaining Theorem 1.4. We prove its finite version 
in $\S 2$, and in $\S 3$ the derivations of Theorems 1.3 and 1.4 are briefly sketched. We also present a "near converse" to Theorem 1.2-that the trace of analytic motion, restricted to any smaller disc $\bar{D}(0, r), r<1$, is the polynomial hull of a subset of $\partial D(0, r) \times \mathbb{C}$ (Proposition 3.3).

\section{HOLOMORPHIC AXIOM OF CHOICE: FINITE CASE}

In this section we prove the following lemma, which asserts that Theorem 1.4 holds for holomorphic motions of finite sets $E$. (We will denote by $H(D)$ the class of holomorphic maps from $D$ to $\mathbb{C}$, and we will let $A(\dot{D})=H(D) \cap C(\bar{D})$.)

Lemma 2.1. Let $f_{0}, f_{1}, \ldots, f_{n} \in H(D), n \geq 1$. Assume that for each $z \in D$ all the values $f_{0}(z), f_{1}(z), \ldots, f_{n}(z)$ are distinct. Then, for every $a \in \mathbb{C}$ such that $a \neq f_{0}(0), f_{1}(0), \ldots, f_{n}(0)$, there is a $g \in H(D)$ such that $g(0)=a$ and, for each $z \in D, g(z) \neq f_{0}(z), f_{1}(z), \ldots, f_{n}(z)$.

To prove this, we construct a special polynomial hull which, as stated above (Theorem 1.2), must admit many analytic discs. One of them will be the graph of the desired map $g$. In fact, there is no need to use the full generality of Theorem 1.2, and we will apply an earlier result of Forstneric [3], which we recall now, for the convenience of the reader.

Notations and Assumptions 2.2 [3]. For $\zeta \in \partial D$ and $0 \leq t \leq 1, M^{t}(\zeta)$ denotes a Jordan curve in $\mathbb{C}$ with fixed homeomorphism onto

$$
s \rightarrow b(s, t, \zeta): R / Z \rightarrow M^{t}(\zeta) .
$$

Assume that:

(i) the map $b: \partial D \times R / Z \times[0,1] \rightarrow \mathbb{C}$ is $C^{k, \alpha}$-smooth with $k \geq 2$ and $\alpha \in(0,1)$;

(ii) $\partial b / \partial s \neq 0$ everywhere;

(iii) $M^{0}(\zeta)=\{|w|=R\}, M^{1}(\zeta)=\{|w|=r\}$, for every $\zeta \in \partial D$, where $r, R$ are fixed numbers such that $0<r<R$; and

(iv) if $0 \leq t_{1}<t_{2} \leq 1$ and $\zeta \in \partial D$, then $M^{t_{2}}(\zeta)$ is contained in the bounded connected component of $\mathbb{C} \backslash M^{t_{1}}(\zeta)$.

Furthermore, we use the following notations: $M^{t}:=\bigcup_{\zeta \in \partial D}\{\zeta\} \times M^{t}(\zeta) ; h\left(M^{t}\right)$ is the polynomial hull of $M^{t} ; S^{t}:=$ the relative topological boundary of $h\left(M^{t}\right)$ in $\bar{D} \times \mathbb{C}$; and $V^{t}:=$ the relative interior of $h\left(M^{t}\right)$ in $\bar{D} \times \mathbb{C}$.

Theorem 2.3 [3]. If tori $M^{t}, 0 \leq t \leq 1$, satisfy Notations and Assumptions 2.2 above, then there is a map

$$
(s, t, z) \rightarrow f(s, t, z): R / Z \times[0,1] \times \bar{D} \rightarrow \mathbb{C},
$$

of class $C^{k-1, \alpha}$, such that

(i) for every $(t, \zeta) \in[0,1] \times \partial D$, the map $s \rightarrow f(s, t, \zeta)$ is a homeomorphism of $R \times Z$ onto $M^{t}(\zeta)$, and $\partial f / \partial s \neq 0$; 
(ii) for every $t \in[0,1]$, the map $(s, z) \rightarrow(z, f(s, t, z))$ is a homeomorphism of $R / Z \times \bar{D}$ onto $S^{t}=\partial_{\bar{D} \times \mathbb{C}} h\left(M^{t}\right)$;

(iii) $t \rightarrow S^{t}, t \in[0,1]$, is a continuous correspondence with respect to the Hausdorff distanc: topology;

(iv) for $t \in[0,1], M^{t} \subset S^{t}, S^{t} \cup V^{t}=h\left(M^{t}\right), S^{t} \cap V^{t}=\varnothing$;

(v) if $0 \leq t_{1}<t_{2} \leq 1$, then $S^{t_{2}} \subset V^{t_{1}}$; in particular, $S^{t_{1}}$ and $S^{t_{2}}$ are disjoint;

(vi) $\bigcup_{0 \leq t \leq 1} S^{t}=\bar{D} \times\{w \in \mathbb{C}: r \leq|w| \leq R\}$; and

(vii) whenever $t \in[0,1]$ and $g \in A(\bar{D})$ are such that $g(z) \neq 0$ for all $z \in \bar{D}$, and $g(\zeta) \in M^{t}(\zeta)$ for every $\zeta \in \partial D$, then there is $s \in R / Z$ such that $g(z) \equiv f(s, t, z)$, for $z \in \bar{D}$.

Comments. Properties (i)-(iv) and (vii) are stated in Forstnerič [3, Theorems $1,3,4]$, and (v) follows from those formulated in [3, p. 886, bottom lines]; only property (vi) may require some explanation. Let $Z=\bigcup_{0 \leq t \leq 1} S^{t}$ and $Z(z)=\{w:(z, w) \in Z\} ;$ then (vi) is equivalent to the relation $Z(\bar{z})=\{w \in$ $\mathbb{C}: r \leq|w| \leq R\}$. By Notations and Assumptions 2.2(iii) and the definition of the polynomial hull,

$$
\begin{array}{cl}
h\left(M^{0}\right)=\bar{D} \times \bar{D}(0, R) ; & h\left(M^{1}\right)=\bar{D} \times \bar{D}(0, r) ; \\
S^{0}=\bar{D} \times \partial D(0, r) ; & S^{1}=\bar{D} \times \partial D(0, r) .
\end{array}
$$

By properties (ii) and (iv) of Theorem 2.3, the map

$$
(s, t) \rightarrow f(s, t, z): R / Z \times[0,1] \rightarrow Z(z)
$$

is one-to-one and, therefore, a homeomorphism of two annuli. In view of (2.1), this homeomorphism maps the boundary of $R / Z \times[0,1]$ onto the boundary of $\{w \in \mathbb{C}: r \leq|w| \leq R\}$, and so the latter set equals $Z(z)$, which proves (vi).

Proof of Lemma 2.1. Applying holomorphic coordinate change $(z, w) \rightarrow$ $\left(z, w-f_{0}(z)\right)$, we can assume without loss of generality that $f_{0} \equiv 0$. We first prove the following assertions.

Assertion 1. Lemma 2.1 holds when $f_{0} \equiv 0$ and $f_{1}, f_{2}, \ldots, f_{n}$ are $C^{k+1}$ smooth on $\bar{D} ; k \geq 2$.

Choose $r>0, R>0$, such that

$$
\begin{aligned}
& r<\min \left\{\left|f_{j}(z)\right|: z \in \bar{D}, j=1, \ldots, n\right\} \\
& R>\max \left\{\left|f_{j}(z)\right|: z \in \bar{D}, j=1, \ldots, n\right\} .
\end{aligned}
$$

Assertion 2. For the chosen values of $r, R$, there is a family of Jordan curves $M^{t}(\zeta), 0 \leq t \leq 1, \zeta \in \partial D$, satisfying all the Notations and Assumptions 2.2, and such that, for some value $t_{0} \in(0,1)$,

$$
\left\{f_{1}(\zeta), f_{2}(\zeta), \ldots, f_{n}(\zeta)\right\} \subset M^{t_{0}}(\zeta), \quad \zeta \in \partial D .
$$


We prove Assertion 2 first. Applying (for example) the Whitney extension theorem [9, Theorem 1.5.6], we can construct a $C^{k}$-smooth complex-valued function $X(\rho, \theta, w)$ defined on $[0.1] \times R \times \mathbb{C}$, such that

$$
\begin{gathered}
X(\rho, \theta+2 \pi, w)=X(\rho, \theta, w), \\
X(\rho, \theta, w)=0 \quad \text { if }|w| \leq r \text { or }|w| \geq R ; \text { and }
\end{gathered}
$$

$$
X\left(\rho, \theta, f_{j}\left(\rho e^{i \theta}\right)\right)=\frac{\partial}{\partial \rho} f_{j}\left(\rho e^{i \theta}\right), \quad 0 \leq \rho \leq 1, \theta \in R, j=1, \ldots, n .
$$

(Function $X$ can easily be constructed directly by the use of the partition-ofunity argument.)

Since $X$ is bounded and uniformly Lipschitz (by conditions (2.3), (2.4), and smoothness), the well-known ODE results [5, Chapter II, Theorem 1.1] imply that the initial-value problem

$$
\left\{\begin{array}{l}
\frac{\partial y}{\partial \rho}=X(\rho, \theta, y(\rho, \theta)), \quad 0 \leq \rho \leq 1, \theta \in R, \\
y(0, \theta)=w_{0}
\end{array}\right.
$$

has a unique solution $\rho \rightarrow y\left(\rho, \theta, w_{0}\right):[0,1] \rightarrow \mathbb{C}$. Furthermore [5, Chapter $\mathrm{V}]$, the function $y\left(\rho, \theta, w_{0}\right)$ is $C^{k}$-smooth on $[0,1] \times R \times \mathbb{C}$. Let $\Phi_{z}(w)=$ $y(\rho, \theta, w)$, where $z=\rho e^{i \theta}, z \in \bar{D}$. Then

$$
\begin{gathered}
\Phi_{z}: \mathbb{C} \rightarrow \mathbb{C} \text { is a } C^{k} \text {-diffeomorphism, } \quad z \in \bar{D}, \\
\Phi_{0}(w)=w, \quad w \in \mathbb{C},
\end{gathered}
$$

and, by (2.5) and the uniqueness of the solution $y$,

$$
\Phi_{z}\left(f_{j}(0)\right)=f_{j}(z), \quad j=0,1,2, \ldots, n, z \in \bar{D} .
$$

We leave without the proof the following assertion:

Assertion 3. There is a $C^{\infty}$-diffeomorphism

$$
(t, s) \rightarrow \Psi(t, s):[0,1] \times R / Z \rightarrow\{r \leq|w| \leq R\},
$$

such that

$$
\begin{gathered}
\Psi\left(\frac{1}{2}, 2 \pi i j / n\right)=f_{j}(0), \quad j=1,2, \ldots, n, \\
\Psi(0, s)=R e^{i s}, \quad \Psi(1, s)=r e^{i s}, \quad s \in R / Z .
\end{gathered}
$$

We can now define the tori $M^{t}$ required in Assertion 2 as follows:

$$
M^{t}(\zeta)=\left\{\Phi_{\zeta}(\Psi(t, s)): s \in R / Z\right\}, \quad 0 \leq t \leq 1, \zeta \in \partial D .
$$

The preceding construction implies that the family of Jordan curves $M^{t}(\zeta)$, equipped with parametrizations $b(s, t, \zeta)=\Phi_{\zeta}(\Psi(t, s))$, satisfies all the Notations and Assumptions 2.2. Furthermore, $f_{j}(\zeta) \in M^{t_{0}}(\zeta), t_{0}=\frac{1}{2}$, because 
$f_{j}(\zeta)=\Phi_{\zeta}\left(f_{j}(0)\right)=\Phi_{\zeta} \Psi\left(\frac{1}{2}, 2 \pi i j / n\right)$, by (2.8) and (2.9), which completes the proof of Assertion 2.

To prove Assertion 1, we apply Forstnerič's Theorem 2.3 to the family $M^{t}(\zeta)$ defined above; the symbols $M^{t}, S^{t}$, and $f(t, s, z)$ have the same meaning as in this theorem. Since $f_{j}(z) \neq 0, z \in \bar{D}$, and $f_{j}(\zeta) \in M^{t_{0}}(\zeta), \zeta \in \partial D$, conditions (vii) and (ii) of Theorem 2.3 imply that

$$
\operatorname{graph}\left(f_{j}\right) \subset S^{t_{0}}, \quad j=1,2, \ldots, n \text {. }
$$

Consider now an arbitrary $a \in \mathbb{C} \backslash\left\{0, f_{1}(0), \ldots, f_{n}(0)\right\}$. If $0<|a|<r$ or $|a|>R$, let $g(z) \equiv a, z \in \bar{D}$. By (2.11) and (vi) of Theorem 2.3, we get $g(z) \neq f_{j}(z), j=0,1, \ldots, n, z \in \bar{D}$.

If $r \leq|a| \leq R$, then by Theorem 2.3(iv) and (vi), there is a unique $t \in[0,1]$, such that $(0, a) \in S^{t}$. By condition (ii) of the same theorem, there is a unique $s \in R / Z$, such that $f(s, t, 0)=a$, and so, if we let $g(z):=f(s, t, z)$, then $g \in A(\bar{D})$, and

$$
\operatorname{graph}(g) \subset S^{t}, \quad g(0)=a, \quad g(z) \neq 0, \quad z \in \bar{D} .
$$

If $t \neq t_{0}$, then $S^{t} \cap S^{t_{0}}=\varnothing$ by Theorem 2.3(iv) and, using (2.11), we get

$$
g(z) \neq f_{1}(z), f_{2}(z), \ldots, f_{n}(z), \quad z \in \bar{D} .
$$

Clearly, $g(z) \neq 0=f_{0}(z)$ by Theorem 2.3(vi). If $t=t_{0}$, the graphs of $g, f_{1}, \ldots, f_{n}$ are analytic discs contained in $S^{t_{0}}$, which are mutually disjoint by conditions (ii) and (vii) of Theorem 2.3. This completes the proof of Assertion 1 and the general case will be reduced to it.

We continue to assume that $f_{0}(z) \equiv 0$, but $f_{1}, \ldots, f_{n}$ are no longer bounded. By applying the additional coordinate change $(z, w) \rightarrow\left(z, w / f_{1}(z)\right)$, we can assume without loss of generality that $f_{0}(z) \equiv 0, f_{1}(z) \equiv 1$, and

$$
f_{j}: D \rightarrow W=\mathbb{C} \backslash\{0,1\}, \quad j=2, \ldots, n .
$$

Fix $a \notin\left\{f_{0}(0), f_{1}(0), \ldots, f_{n}(0)\right\}$; in particular $a \in W$. We can apply Assertion 1 to every closed disc $\bar{D}_{\rho}=\{|z| \leq \rho\}$ and obtain, for every $0<\rho<1$, an analytic function $g^{\rho}: D_{\rho} \rightarrow \mathbb{C}$ such that

$$
g^{\rho}(z) \neq f_{0}(z), \ldots, f_{n}(z), \quad z \in D_{\rho} ; g^{\rho}(0)=a .
$$

Since, in particular, $g^{\rho}\left(D_{\rho}\right) \subset W, \rho \in(0,1)$, we can apply now the wellknown fact that $W=\mathbb{C} \backslash\{0,1\}$ is completely hyperbolic [7, Chapter I, §3].

Consider an arbitrary $\rho_{0} \in(0,1)$, and fix $\rho_{1} \in\left(\rho_{0}, 1\right)$. It follows from the complete hyperbolicity of $W$ that there is a compact subset $K \subset W$ such that $K \supset g^{\rho}\left(\bar{D}_{\rho_{0}}\right), \rho>\rho_{1} . K$ can be taken to be the closed hyperbolic ball in $W$ with center at $a$, of sufficiently large radius [7, Chapter I, Proposition 3.1]. Hence $\left\{g^{\rho} \mid D_{\rho_{0}}\right\}_{\rho>\rho_{1}}$ is a Montel family whenever $\rho_{0}<\rho_{1}<1$, and so there is a sequence $\rho(n)<1$ such that $g^{\rho(n)}$ converges uniformly on compact subsets of $D$ to some $g \in H(D)$. Clearly, $g(0)=a$. Since $g^{\rho(n)}(z) \neq f_{j}(z)$ 
for $|z|<\rho_{1}<\rho(n)$ and $g(0) \neq f_{j}(0)$, Hurwitz's theorem implies that $g(z) \neq$ $f_{0}(z), \ldots, f_{n}(z)$. Q.E.D.

\section{APPLICATIONS OF THE HOLOMORPHIC AXIOM OF CHOICE}

We first show how the finite case of the holomorphic axiom of choice implies the general case (Theorem 1.4) and the extendability of holomorphic notions (Theorem 1.3). Since these derivations have already been indicated by Sullivan and Thurston [13, §1], we give only a brief sketch, for the convenience of the reader. Afterward we discuss further relations between polynomial hulls and holomorphic motions.

Proof of Theorem 1.4 (sketch). Choose in $E$ a dense sequence $\left\{e_{n}\right\}_{n=0}$ of mutually distinct points ( $E$ is assumed to be infinite). By replacing motion $f(z, w)$ by

$$
(z, w) \rightarrow\left(f(z, w)-f\left(z, e_{0}\right)\right) /\left(f\left(z, e_{1}\right)-f\left(z, e_{0}\right)\right),
$$

we can assume, without loss of generality, that

$$
f\left(z, e_{0}\right) \equiv 0, \quad f\left(z, e_{1}\right) \equiv 1, \quad z \in D .
$$

Let $a \notin E$. Applying Lemma 2.1 to functions $f_{0}, f_{1}, \ldots, f_{n}, n \geq 2$, where $f_{n}(z)=f_{n}\left(z, e_{n}\right)$, and in particular $f_{0} \equiv 0, f_{1} \equiv 1$, we obtain $g_{n} \in H(D)$, such that $g(0)=a$ and $g_{n}(a) \notin\left\{0,1, f_{2}(z), \ldots, f_{n}(z)\right\}$. Since $g_{n}(D) \subset$ $W=\mathbb{C} \backslash\{0,1\}$, the same application of the complete hyperbolicity of $W$ as made at the end of $\S 2$ yields that $\left\{g_{n}\right\}$ is a normal family and, since $g_{n}(0)=a$, $n \geq 1$, functions $g_{n}$ converge uniformly on compact subsets of $D$ to some $g \in H(D)$. Clearly, $g(0)=a$. Suppose that $g\left(z_{0}\right)=f\left(z_{0}, w_{0}\right)$, for some $w_{0} \in E$. For some subsequence $e_{n_{k}}, \lim e_{n_{k}}=w_{0}$ and the nowhere-vanishing functions $f_{n_{k}}-g$ converge uniformly on compact subsets of $D$ to a function vanishing at $z_{0}$, but not at 0 , which contradicts Hurwitz's theorem. Hence $g(z) \notin f_{z}(E)$. Q.E.D.

Proof of Theorem 1.3 (sketch). The argument is essentially the same and was also indicated by Sullivan and Thurston $[13, \S 1]$. Choose a countable dense sequence $\left\{a_{n}\right\}_{n=1}^{\infty}$ in $\mathbb{C} \backslash E$ with mutually distinct terms $a_{n}$. By Theorem 1.4 , there is a function $g_{1} \in H(D)$ such that $g_{1}(0)=a_{1}$ and $g_{1}(z) \notin f_{z}(E)$. We will construct inductively a sequence of functions $g_{2}, \ldots, g_{n}, \ldots \in H(D)$ such that $g_{n}(0)=a_{n}$, and $g_{n}(z), n=1,2, \ldots$ are mutually distinct points of $\mathbb{C} \backslash f_{z}(E)$. If $g_{1}, g_{2}, \ldots, g_{n}$ are already constructed, then $f(z, w), g_{1}(z), \ldots, g_{n}(z)$ define together a holomorphic motion over $D$, of the set $E \cup\left\{a_{1}, a_{2}, \ldots, a_{n}\right\}$. Applying the holomorphic principle of choice (Theorem 1.4) to this new motion and letting $a:=a_{n+1}$, we obtain $g_{n+1} \in H(D)$ such that $g_{n+1}(0)=a_{n+1}$ and $g_{n+1}(z) \notin f_{z}(E) \cup\left\{g_{1}(z), \ldots, g_{n}(z)\right\}, z \in D$.

If we let $E^{*}=E \cup\left\{a_{n}: n=1,2, \ldots\right\}$, define $f^{*}: D \times E^{*} \rightarrow \mathbb{C}$ by $f^{*}(z, w)=f(z, w)$ when $z \in E$, and let $f^{*}\left(z, e_{n}\right)=g_{n}(z)$, then we obtain a holomorphic motion over $D$ of the nonclosed, dense set $E^{*}$ which, by the "lambda lemma" (Lemma 1.1) has unique extension to an analytic motion $F: D \times \mathbb{C} \rightarrow \mathbb{C}$. This is the required extension of $f$. Q.E.D. 
Using extensions of holomorphic motions, we can slightly improve Theorem 1.2 as follows:

Corollary 3.1. Let $X=\bigcup_{\zeta \in \partial D}\{\zeta\} \times X(\zeta) \subset \mathbb{C}^{2}$ be a compact set with all the fibers $X(\zeta)$ connected. Assume that the polynomial hull $h(X)$ intersects $D \times \mathbb{C}$, and denote by $E$ the fiber over $0 ; E=\{w:(0, w) \in h(X)\}$. Then there exists a holomorphic motion $f: D \times E \rightarrow \mathbb{C}$ of the nonempty set $E$ such that $h(X) \cap D \times \mathbb{C}$ is equal to the trace of $E$; i.e., $h(X) \cap D \times \mathbb{C}=\{(z, f(z, w)): z \in D, w \in E\}$. Proof. Let $S=\partial h(X) \cap D \times \mathbb{C} ; V=\operatorname{Int} h(X) \cap D \times \mathbb{C}$. Since $S$ is covered by mutually disjoint analytic discs [12, Theorem 1.1$]$, for very $w \in S(0)$, there is a unique analytic disc passing through $w$, which is the graph of a function $g_{w} \in H(D)$, such that $g_{w}(0)=w$. Clearly, the function $g(z, w)=g_{w}(z)$, $(z, w) \in D \times S(0)$, defines a holomorphic motion which, by Theorem 1.3, has an extension to a holomorphic motion of $\mathbb{C}$; i.e., $G: D \times \mathbb{C} \rightarrow \mathbb{C}$. We claim that $G(D \times E)=h(X) \cap(D \times \mathbb{C})$.

Denote by $E(z), S(z), V(z)$ the fibers, over $z \in D$, of $h(X), S, V$, respectively. By [12, Theorem 1.1(b), (c)], $E(z)=S(z) \cup V(z)$ and $S(z)=$ $\partial_{\mathbb{C}} E(z)$. Since (by Lemma 1.1) every $G_{z}(\cdot)=G(z, \cdot)$ is a homeomorphism of $\mathbb{C}$ onto itself, and since $G_{z}(S(0))=S(z)$, we conclude that $G_{z}(E)=E(z)$ (note that $E=E(0)$ ); that is, $G(D \times E)=h(X) \cap D \times \mathbb{C}$. We complete the proof by letting $f=G \mid D \times E$. Q.E.D.

It is a natural question whether the converse of Corollary 3.1 holds. Namely, if $f: D \times E \rightarrow \mathbb{C}$ is a bounded holomorphic motion of (say, connected) compact set $E$, can we represent its trace as follows?

$$
\{(z, f(z, w)): z \in D, w \in E\}=h(X) \cap(D \times \mathbb{C}),
$$

where $X$ is some compact subset of $\partial D \times \mathbb{C}$. It turns out that this can fail even when $E$ is a single point.

Example 3.2. Choose a Blaschke product $B(z)$ such that each point of $\partial D$ is a singular point of $B(\cdot)$. Let $E=\{B(0)\}$, and define $f: D \times E \rightarrow \mathbb{C}$ by $f(z, B(0))=B(z)$. Clearly, if (3.1) holds for some $X$, it must be true with $X=\{(\zeta, w):|\zeta|=1, w \in \mathrm{Cl}(H, \zeta)\}$, where $\mathrm{Cl}(B, \zeta)$ denotes the cluster set of $B$ at $\zeta$. By [4, Chapter II, Theorem 6.7], $\mathrm{Cl}(B, \zeta)=\bar{D}$; thus $X=\partial D \times \bar{D}$ and $h(X)=\bar{D} \times \bar{D}$, so (3.1) fails.

In this example, failure of representation (3.1) was caused by bad boundary properties of $f(z, w)$ as $|z| \nearrow 1$. Mild boundary regularity assumptions likely should suffice for (3.1) to hold; the following fact is a first step in this direction.

Proposition 3.3. Let $f: D \times E \rightarrow \mathbb{C}$ be a bounded holomorphic motion; i.e., $\sup \{|f(z, w)|: z \in D, w \in E\}<+\infty$ of the compact, simply connected set $E$. Then, for every $\rho<1$,

$$
\{(z, f(z, w)):|z| \leq \rho, w \in E\}=h\left(X_{\rho}\right),
$$

where $X_{\rho}=\left\{\left(\rho e^{i \theta}, f\left(\rho e^{i \theta}, w\right)\right): \theta \in R, w \in E\right\}$. 
Proof. Let $F: D \times \mathbb{C} \rightarrow \mathbb{C}$ be a holomorphic motion, extending $f$, as in Theorem 1.3. Suppose that (3.2) fails, and denote the left-hand side of (3.2) by $Z_{\rho}$. Thus, there is a $\left(z_{0}, w_{0}\right) \in D \times(\mathbb{C} \backslash E)$ such that $\left(z_{0}, F\left(z_{0}, w_{0}\right)\right) \in h\left(X_{\rho}\right) \backslash Z_{\rho}$. Let $w(t), 0 \leq t \leq 1$, be a continuous arc contained in $(\mathbb{C} \backslash E) \cup\{\infty\}$ and joining $w_{0}$ to $\infty$. Then $N_{t}=\left\{(z, F(z, w(t))): z \in D_{\rho}\right\}, t \in[0,1]$, are relatively closed analytic discs in $D_{\rho} \times \mathbb{C}$ such that for every $t_{0}>0$, the sets $\bigcup_{t \geq t_{0}} \partial N_{t}$ and $X_{\rho}$ are closed and disjoint, while $N_{0}$ intersects $h\left(X_{\rho}\right), \bar{N}_{t} \cap h\left(X_{\rho}\right)=\varnothing$ for $t$ close to 1 , and the correspondence $t \rightarrow \bar{N}_{t}$ is continuous in Hausdorff topology. This would imply that the set $D_{\rho} \times \mathbb{C} \backslash h\left(X_{\rho}\right)$ does not have the Kontinuitatsatz property, and so is not pseudoconvex, contrary to what was established in [10, Theorem 2.1]. Namely the complement in $\mathbb{C}^{2}$ of a set satisfying the local maximum principle is locally pseudoconvex, which applies to the polynomial hull $h\left(X_{\rho}\right)$ by Rossi's local maximum modulus principle. (See also Kumagai [6, §1, Theorems 2, 3] for a similar argument.) Q.E.D.

\section{REFERENCES}

1. H. Alexander and J. Wermer, Polynomial hulls with convex fibers, Math. Ann. 271 (1985), 99-109.

2. L. Bers and H. L. Royden, Holomorphic families of injections, Acta Math. 157 (1986), 259-286.

3. F. Forstnerič, Polynomial hulls of sets fibered over the circle, Indiana Univ. Math. J. 37 (1988), 869-889.

4. J. B. Garnett, Bounded analytic functions, Academic Press, New York, 1981.

5. P. Hartman, Ordinary differential equations, John Wiley and Sons, New York, 1964.

6. D. Kumagai, Variations of fibers and polynomially convex hulls, Complex Variables 11 (1989), 261-267.

7. S. Lang, Introduction to complex hyperbolic spaces, Springer-Verlag, New York, 1987.

8. R. Mañé, P. Sad and D. Sullivan, On the dynamics of rational maps, Ann. Sci. École Norm. Sup. 16 (1983), 193-217.

9. R. Narasimhan, Analysis on real and complex manifolds, North-Holland, Amsterdam, 1968.

10. Z. Slodkowski, Analytic set-valued functions and spectra, Math. Ann. 256 (1981), 363-386.

11. _ Polynomial hulls with convex sections and interpolating spaces, Proc. Amer. Math. Soc. 96 (1986), 255-260.

12. _ Polynomial hulls in $\mathbb{C}^{2}$ and quasicircles, Annali Scuola Norm. Sup. (Pisa), Ser. 416 (1989), 367-391.

13. D. P. Sullivan and W. P. Thurston, Extending holomorphic motions, Acta Math. 157 (1986), 243-257.

Department of Mathematics, University of Illinois at Chicago, Chicago, Illinois 60680 